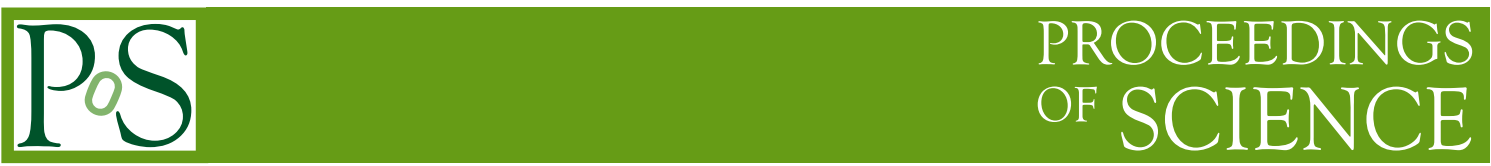

\title{
Search for New Physics with the SHiP experiment at CERN
}

\author{
Sergey Shirobokov ${ }^{a, *}$ on behalf of the SHiP collaboration \\ amperial College London, \\ Blackett Laboratory, London, United Kingdom \\ E-mail: s.shirobokov17@imperial.ac.uk
}

\begin{abstract}
The SHiP Collaboration has proposed a general-purpose experimental facility operating in beam dump mode at the CERN SPS accelerator with the aim of searching for light, long-lived exotic particles. The detector system aims at measuring the visible decays of hidden sector particles to both fully reconstructible final states and to partially reconstructible final states with neutrinos, in a nearly background free environment. In addition to that, it can detect light dark matter via its scattering and study tau neutrino physics. Using a high-intensity beam of $400 \mathrm{GeV}$ protons, the experiment is capable of integrating $2 \times 10^{20}$ protons in five years, which allows probing dark photons, dark scalars, axion-like particles and heavy neutral leptons with $\mathrm{GeV}$-scale masses at sensitivities that exceed by orders of magnitude those of existing and projected experiments. The sensitivity to heavy neutrinos will allow for the first time to probe, in the mass range between the kaon and the charm meson mass, a coupling range for which baryogenesis and the magnitude of the active neutrino masses can be explained. The sensitivity to light dark matter reaches well below the elastic scalar dark matter relic density limits in the range from a few $\mathrm{MeV} / \mathrm{c}^{2}$ up to 200 $\mathrm{MeV} / \mathrm{c}^{2}$.
\end{abstract}

40th International Conference on High Energy physics - ICHEP2020

July 28 - August 6, 2020

Prague, Czech Republic (virtual meeting)

${ }^{*}$ Speaker 


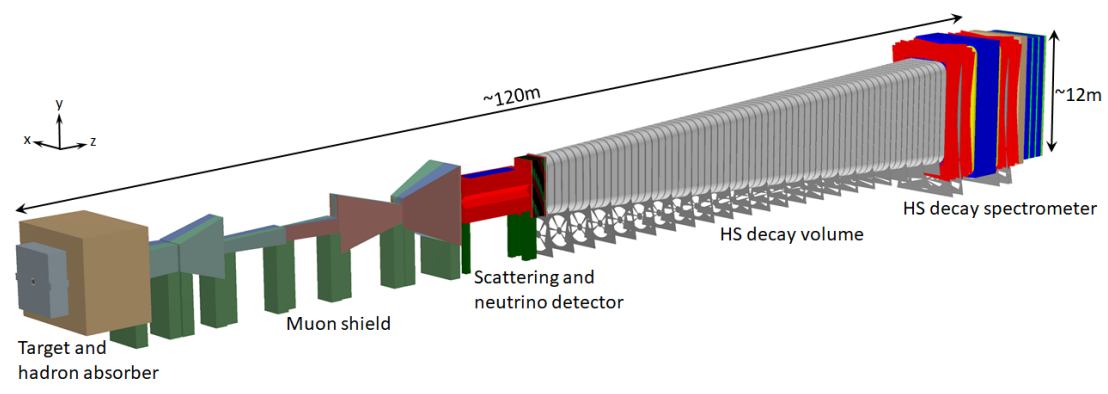

Figure 1: Overview of the SHiP experiment as implemented in the full simulation.

\section{Introduction}

The Search for Hidden Particles (SHiP) experiment has been proposed to study a wide variety of beyond Standard Model (SM) models, containing weakly interacting long-lived particles (wLLPs) in the mass range of the $O(10) \mathrm{GeV}[1,2]$. There are two key factors required to perform such study: high production rates of heavy flavour mesons, such as charm and beauty mesons, which subsequently decay to wLLPs, and a zero background environment.

To achieve the first goal a dedicated Beam Dump Facility (BDF) at the SPS is being developed [3]. To obtain an optimal heavy flavour production cross-section, the BDF will deliver protons with an energy of $400 \mathrm{GeV}$, slowly extracted from the SPS with one second long spills. The nominal beam intensity is projected to be $4 \times 10^{13}$ protons on target (POT) per spill. This will result in $4 \times 10^{19}$ POT per year for the experiment, while respecting LHC and HL-LHC requirements as well as other physics programs at the CERN North Area. SHiP is expected to achieve $2 \times 10^{20}$ POT in five years of operation, which will result in $10^{18} \mathrm{D}, 10^{14} \mathrm{~B}$ meson and $10^{16} \tau$ leptons [4].

To achieve zero background, SHiP will exploit the slow extraction of the beam from the SPS, that allows it to control combinatorial background. Should the observation require confirmation, the beam can be bunched in order to increase the discrimination between Light Dark Matter (LDM) and background utilising particle time of flight [4]. SHiP will have a dedicated muon shield to sweep away muons originating in the target, a decay volume under vacuum and surrounded by veto taggers, a timing detector, and a magnetic spectrometer.

\section{The SHiP detector}

The current layout of the SHiP experiment is presented in Figure 1. It starts with a dense, $12 \lambda_{\text {int }}$-long target [5], followed by a hadron stopper and an active muon shield. A short $\lambda_{\text {int }}$ allows the target to absorb pions and kaons before their decay, while the hadron stopper absorbs electromagnetic radiation and hadrons emerging from the target. A magnetic coil, embedded in hadron stopper serves as a first section of the active muon shield.

Since the muon shield is a crucial part of the experiment, it has undergone several rounds of optimisation utilising machine learning methods [6,7]. The goal of the optimisation was to maximise physics performance, while minimising shield length and cost. The resulting muon shield is capable of reducing the flux of muons emerging from the target by six orders of magnitude 
in the detector acceptance. However, it must be noted, that dedicated $R \& D$ is required to validate simulation results and the design's robustness.

The SHiP experiment comprises two complementary detectors. The first detector, the scattering neutrino detector (SND), is located downstream of the muon shield and is optimised for scattering signatures of LDM and for $\tau$ neutrino physics. It is equipped with an emulsion based spectrometer and target trackers, enclosed inside a magnetised volume. Due to the magnetisation, it will be capable of distinguishing between (anti) $v_{\mu}, v_{\tau}$ and provide first direct observation of $\bar{v}_{\tau}$.

The second detector, called the Hidden Sector (HS) detector, is located after the decay volume, and aims to measure the decays of wLLPs to partially and fully reconstructible final states, originating in the $50 \mathrm{~m}$ long decay volume. To eliminate the background while maximising detector acceptance, the decay volume has a pyramidal frustum shape, has a pressure $<10^{-2}$ bar, and is surrounded by liquid scintillator background taggers. The taggers identify neutrino and muon induced inelastic interactions in the material of the SND and in the decay volume walls, which may produce long lived neutral particles, decaying in the decay volume and mimicking HS signal events. It is followed by the $5 \times 10 \mathrm{~m}^{2}$ straw tracker spectrometer and a magnet with a total field integral of $0.65 \mathrm{Tm}$. With the resolution of $\left(\sigma_{p} / p\right)^{2}=(0.5 \%)^{2}+(0.02 \% /[\mathrm{GeV} / \mathrm{c}])^{2} p^{2}$ it is able to accurately reconstruct momenta, decay vertex, mass and impact parameter of the hidden particle at the proton target.

Further downstream, the ECAL, timing detector (TD) and muon system are located. The ECAL consists of two physically separated lead sampling calorimeters, each with a high spatial resolution layer. This construction allows the ECAL to achieve unprecedented resolution of $\sigma_{\theta} \approx$ mrad and provides the capability of reconstructing the decay of axion-like particles to two photons. The TD with a resolution of $\sigma_{t} \approx 100 \mathrm{ps}$ provides a measurement of time-of-coincidence to reject muon combinatorial background. Finally, muon detectors, located downstream, allows full flavour identification of hidden particle decay products.

The overall characteristics of the SND and HS detector are optimised to make SHiP a zero background wLLP discovery experiment.

\section{Physics performance}

Signal sensitivities: SHiP is sensitive to the decays of Heavy Neutral Leptons (HNLs), Dark Photons (DP), Dark Scalars (DS) and axion-like particles (ALPs). Moreover, due to the SND detector SHiP can also detect LDM. Some sensitivities, corresponding to the projected intensity of $2 \times 10^{20}$ POT in five years of operation, obtained with SHiP full MC simulation framework FairShip are presented in Figure $2[8,9]$. It is interesting to note that SHiP is also capable of distinguishing between Majorana and Dirac types of HNLs by detecting lepton flavour violation decays [10].

Background studies: Since SHiP is designed to be a zero background experiment, detailed studies of the background sources have been performed and selection criteria common to all HS searches have been devised. There are two main signatures for HS searches in SHiP: fully and partially reconstructed decays. Example of the former is ALP $\rightarrow \gamma \gamma$ and of the latter $\mathrm{HNL} \rightarrow \mu^{ \pm} \mu^{\mp} \nu$, where for this category there should be at least two charged particles and one neutrino in the final state. In both cases, the signature is an isolated vertex, pointing backwards to the target. The common criteria include cuts on the track momentum, vertex quality, the distance of closest approach of the 


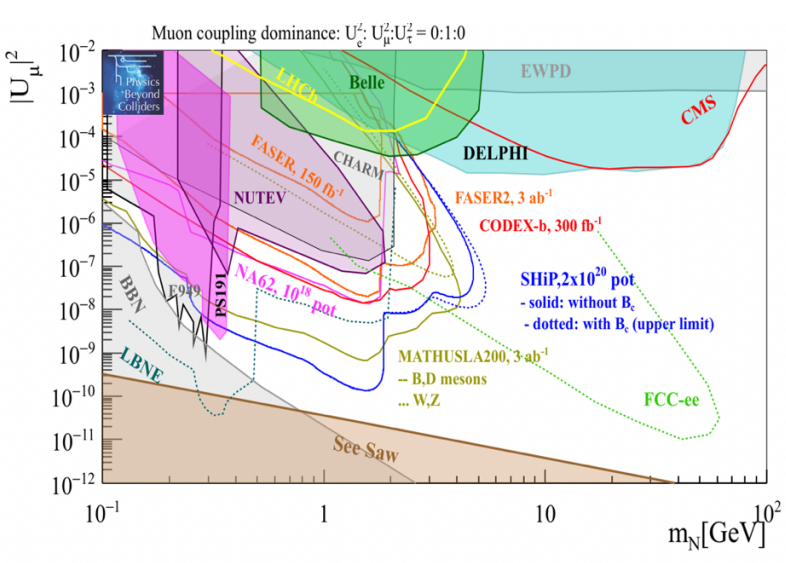

(a)

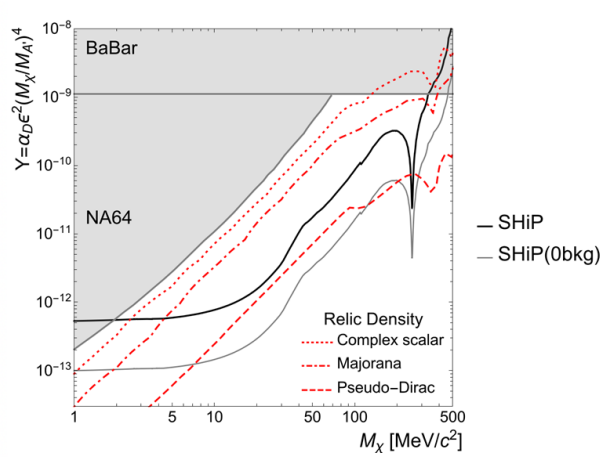

(b)

Figure 2: SHiP's sensitivity to: a) Heavy Neutral Leptons b) Light Dark Matter.

tracks and the impact parameter w.r.t to the target. There are three main sources of background: muon combinatorial, muon inelastic scattering and neutrino deep inelastic scattering. Background from cosmic muons has been proved to be negligible.

Muon combinatorial: Using the muon shield and slow beam extraction it is possible to reduce the initial muon flux to a rate of just $30 \mathrm{kHz}$. Even at this rate, after applying the selection cuts, SHiP is expecting to have $10^{5}$ pairs of muon tracks in five years of operation. However, using SHiP's dedicated timing detector, these can be suppressed to the level of $10^{-2}$ pairs over five years by requiring muons to fall within a $340 \mathrm{ps}$ window, which is three times the resolution of the TD. Further suppression of the background can be done using veto taggers. Combinatorial muons corresponding to one spill, have been generated to study this background. In addition, a dedicated muon flux measurement has been performed by the collaboration to validate the muon flux, which is described below.

Muon inelastic: Based on simulations corresponding to five years of data taking, it was estimated that there will be around $2 \times 10^{8}$ deep inelastic scattering (DIS) of muons on the decay volume walls and experimental hall infrastructure. However, applying selection criteria and background tagger information, this number is reduced to just $6 \times 10^{-4}$ in five years of operation.

Neutrino deep inelastic: DIS scattering of neutrinos in the decay volume walls will produce around $3.5 \times 10^{7} V_{0}$ particles that can mimic the HS signatures. By applying selection cuts and background tagger information, this background is reduced to 0.1 events for fully reconstructed events and 0.3 events for partially reconstructed. DIS scattering off of the air inside the decay volume was estimated to produce $10^{-2}$ events due to the vacuum inside the vessel. The DIS on the experimental cavern walls and floor was found to be negligible. The above calculations have been performed on simulated data corresponding to ten years of data taking.

Neutrino background for LDM: The background for LDM searches via its scattering off of electrons in the SND consists of various types of neutrino scattering in the SND detector. The dominant background comes from topologically indistinguishable neutrino elastic scattering off of electrons or protons and quasi-elastic scattering off of an electron, with an unidentified soft outgoing proton. The total background from all types of neutrino scattering after applying geometric, kinematic and topological cuts was estimated to be 230 events in five years [11]. 


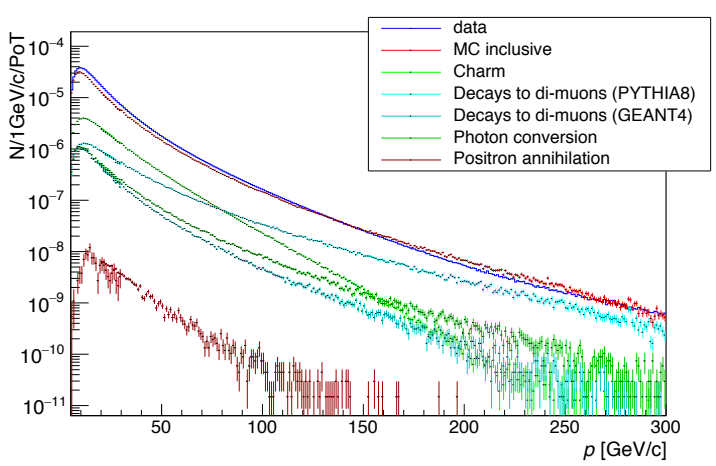

(a)

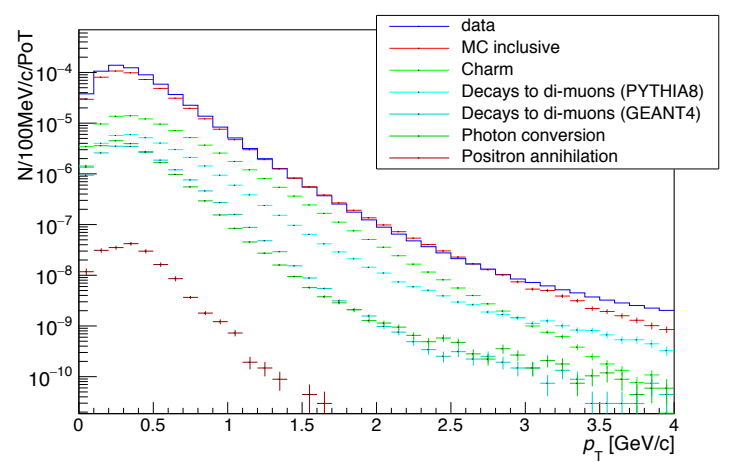

(b)

Figure 3: Measured muon momentum a) and transverse momentum b) distributions from data and simulation. The distributions are normalised to the number of POT. Production channels are shown separately.

\section{Muon flux measurement}

Since muons constitute the major background for the experiment and their flux enters linearly into the estimation of DIS events and quadratically into that of the combinatorial background, a dedicated test beam experiment was performed in summer 2018 at the $\mathrm{H} 4400 \mathrm{GeV} / \mathrm{c}$ proton beam-line of the CERN SPS [12]. A target replica with the full length of the SHiP target was exposed, which allowed the collaboration to measure the cascade production of particles, decaying into muons. About $3.3 \times 10^{11}$ POT have been collected, which accounts to $1 \%$ of the SHiP spill.

The results of the test beam are presented in Figure 3a,b. Considering the complexity of simulating the production mechanisms of muons in the dense target, one can see that there is a good agreement between MC simulation and the observed flux [13].

\section{Charm production in the SHiP target}

The same H4 beam-line was used to collect $1.5 \times 10^{6}$ POT to measure the associated charm production within the thick target [14]. The cascade production of charm plays a crucial role in an accurate estimation of HS particles, possibly produced in charm decays as well as tau neutrino flux. Emulsion Cloud Chamber technology in combination with electronic detectors allows for identification of the primary proton interaction vertex and its multiplicity in a dense environment.

The analysis of the data shows a good agreement between Monte Carlo estimations and data for the vertex position along the beam axis and number of charged tracks, which is a key prerequisite for the next step of the analysis. In addition to that, the experiment helped to develop and test dedicated software and analysis tools, crucial for further steps.

\section{Conclusion}

In this report the key components of the SHiP experiment, as well as estimation of background and signal sensitivity have been described. Up to date results from the muon flux measurement and the charm-production analysis have been presented. These dedicated test beam experiments give confidence in the reliability of the SHiP simulation software. Future R\&D activities of the SHiP Collaboration will be carried out in conjunction with the feasibility studies for the SPS Beam Dump Facility. 


\section{References}

[1] S. Alekhin et al. A facility to Search for Hidden Particles at the CERN SPS: the SHiP physics case. (CERN-SPSC-2015-017. SPSC-P-350-ADD-1), Apr 2015. doi: 10.1088/0034-4885/79/12/124201. URL http: //cds . cern. ch/record/2007686.

[2] The SHiP collaboration. A Facility to Search for Hidden Particles (SHiP) at the CERN SPS. Technical Report CERN-SPSC-2015-016. SPSC-P-350. arXiv:1504.04956, CERN, Geneva, Apr 2015. URL https://cds. cern. ch/record/2007512. Technical Proposal.

[3] C. C. Ahdida et al. SPS Beam Dump Facility - Comprehensive Design Study. Technical report, Dec 2019. URL https: //cds. cern.ch/record/2703984.

[4] The SHiP collaboration. SHiP Experiment - Comprehensive Design Study report. Technical Report CERN-SPSC-2019-049. SPSC-SR-263, CERN, Geneva, Dec 2019. URL https: //cds . cern.ch/ record/2704147.

[5] E. Lopez Sola et al. Design of a high power production target for the beam dump facility at cern. Phys. Rev. Accel. Beams, 22:113001, Nov 2019. doi: 10.1103/PhysRevAccelBeams.22.113001. URL https://link.aps.org/doi/10.1103/PhysRevAccelBeams.22.113001.

[6] A. Baranov et al. Optimising the Active Muon Shield for the SHiP Experiment at CERN. Journal of Physics: Conference Series, 934:012050, dec 2017. doi: 10.1088/1742-6596/934/1/012050. URL https: //doi .org/10 . 1088\%2F1742-6596\%2F934\%2F1\%2F012050.

[7] O. Lantwin. Optimisation of the SHiP experimental design, 2019. URL https://cds . cern. ch/ record/2693177.

[8] The SHiP collaboration. Sensitivity of the ship experiment to heavy neutral leptons. Journal of High Energy Physics, 2019, 04 2019. doi: 10.1007/JHEP04(2019)077. URL https : //doi . org/10 . 1007/ JHEP04 (2019) 077.

[9] J. Beacham et al. Physics beyond colliders at CERN: beyond the Standard Model working group report. Journal of Physics G: Nuclear and Particle Physics, 47(1):010501, Dec 2019. ISSN 1361-6471. doi: 10.1088/1361-6471/ab4cd2. URL http: //dx . doi . org/10 . 1088/1361-6471/ab4cd2.

[10] J.-L. Tastet and I. Timiryasov. Dirac vs. Majorana HNLs (and their oscillations) at SHiP. Journal of High Energy Physics, 2020(4), Apr 2020. ISSN 1029-8479. doi: 10.1007/jhep04(2020)005. URL http: //dx . doi .org/10.1007/JHEP04(2020)005.

[11] The SHiP Collaboration. Sensitivity of the SHiP experiment to light dark matter. arXiv e-prints, art. arXiv:2010.11057, October 2020. URL https: //arxiv . org/abs/2010 . 11057.

[12] The SHiP Collaboration. Muon-flux measurements for SHiP at H4. Technical Report CERNSPSC-2017-020. SPSC-EOI-016, CERN, Geneva, Jun 2017. URL https : //cds . cern . ch/record/ 2267770.

[13] The SHiP collaboration. Measurement of the muon flux from $400 \mathrm{GeV} / \mathrm{c}$ protons interacting in a thick molybdenum/tungsten target. Eur. Phys. J. C, 80(3):284. 11 p, 2020. doi: 10.1140/epjc/ s10052-020-7788-y. URL http://cds. cern. ch/record/2715745.

[14] The SHiP Collaboration. Measurement of associated charm production induced by $400 \mathrm{GeV} / \mathrm{c}$ protons. Technical Report CERN-SPSC-2017-033. SPSC-EOI-017, CERN, Geneva, Oct 2017. URL http: //cds. cern. ch/record/2286844. 